\title{
Electrochemical-assisted photodegradation of mixed dye and textile effluents using TiO2 thin films
}

\begin{abstract}
Mixed dye consists of six commercial dyes and textile effluents from cotton dyeing process were treated by electrochemical-assisted photodegradation under halogen lamp illumination. Two types of effluents were collected which are samples before and after undergone pretreatment at the factory wastewater treatment plant. The photodegradation process was studied by evaluating the changes in concentration employing UV-vis spectrophotometer (UV-vis) and total organic carbon (TOC) analysis. The photoelectrochemical degradation of mixed dye was found to follow the Langmuir Hinshelwood pseudo-first order kinetic while pseudo-second order kinetic model for effluents by using TOC analyses. The chemical oxygen demand (COD) and biochemical oxygen demand (BOD) values of mixed dye and raw effluents were reported. Photoelectrochemical characteristic of pollutants was studied using the cyclic voltammetry technique. Raw effluent was found to exhibit stronger reduction behaviour at cathodic bias potential but slightly less photoresponse at anodic bias than mixed dye.
\end{abstract}

Keyword: Electrochemical-assisted, Photodegradation, Mixed dye, Textile effluents, TiO2 